\title{
Vibrational modes of adsorbed atoms
}

\author{
W. R. Lawrence* and R. E. Allen \\ Department of Physics, Texas A\&M University, College Station, Texas 77843 \\ (Received 1 June 1976)
}

The vibrational spectra have been calculated for seven model adsorbate-substrate systems.

\section{INTRODUCTION}

Recently, ${ }^{1}$ we reported calculations of heat capacities of adsorption for seven model adsorbatesubstrate systems $-{ }^{4} \mathrm{He}$ on $\mathrm{Xe}, \mathrm{Ne}$ on $\mathrm{Ar}, \mathrm{Kr}$, and $\mathrm{Xe}, \mathrm{Ar}$ on $\mathrm{Kr}$ and $\mathrm{Xe}$, and $\mathrm{Kr}$ on $\mathrm{Xe}$, with the substrate having a (100) face and the adsorbed atoms going down in registry with the substrate. We thought that there might be some interest in a detailed description of the vibrational spectra of these systems, since previous treatments of the vibrational modes of adsorbates have involved mass-defect models, ${ }^{2}$ so we have given such a description in the present paper. The method of calculation and details of the model are the same as in Ref. 1 ; in particular, the expansion at the surface due to zero-point vibrations (important for ${ }^{4} \mathrm{He}$ and $\mathrm{Ne}$ adsorbates) is included.

\section{I. PHONON-DISPERSION CURVES}

In Figs. 1-7, the dimensionless frequency $\omega^{*}$ is plotted as a function of the two-dimensional wave vector $\bar{q}$ along the symmetry lines ${ }^{3} \bar{\Gamma} \bar{X}, \bar{X} \bar{M}$, and $\bar{M} \bar{\Gamma} ; \omega^{*}$ is related to the frequency $\omega$ by $\omega^{*}$ $\equiv\left(m_{s} \sigma_{s}^{2} / \epsilon_{s}\right)^{1 / 2} \omega$, where $m_{s}, \sigma_{s}$, and $\epsilon_{s}$ are the atomic-mass and Lennard-Jones-potential parameters for the substrate. These results are for 11 layer slabs with nine layers of substrate sandwiched between two adsorbate layers. We have labeled each surface mode by a number which indicates the plane in which its amplitude is largest (with the plane of adsorbed atoms represented by 1 , the first plane of substrate atoms labeled by 2 , etc.) and by letters which indicate the dominant polarization (with $L$ representing longitudinal polarization, $H$ representing "horizontal" polari-

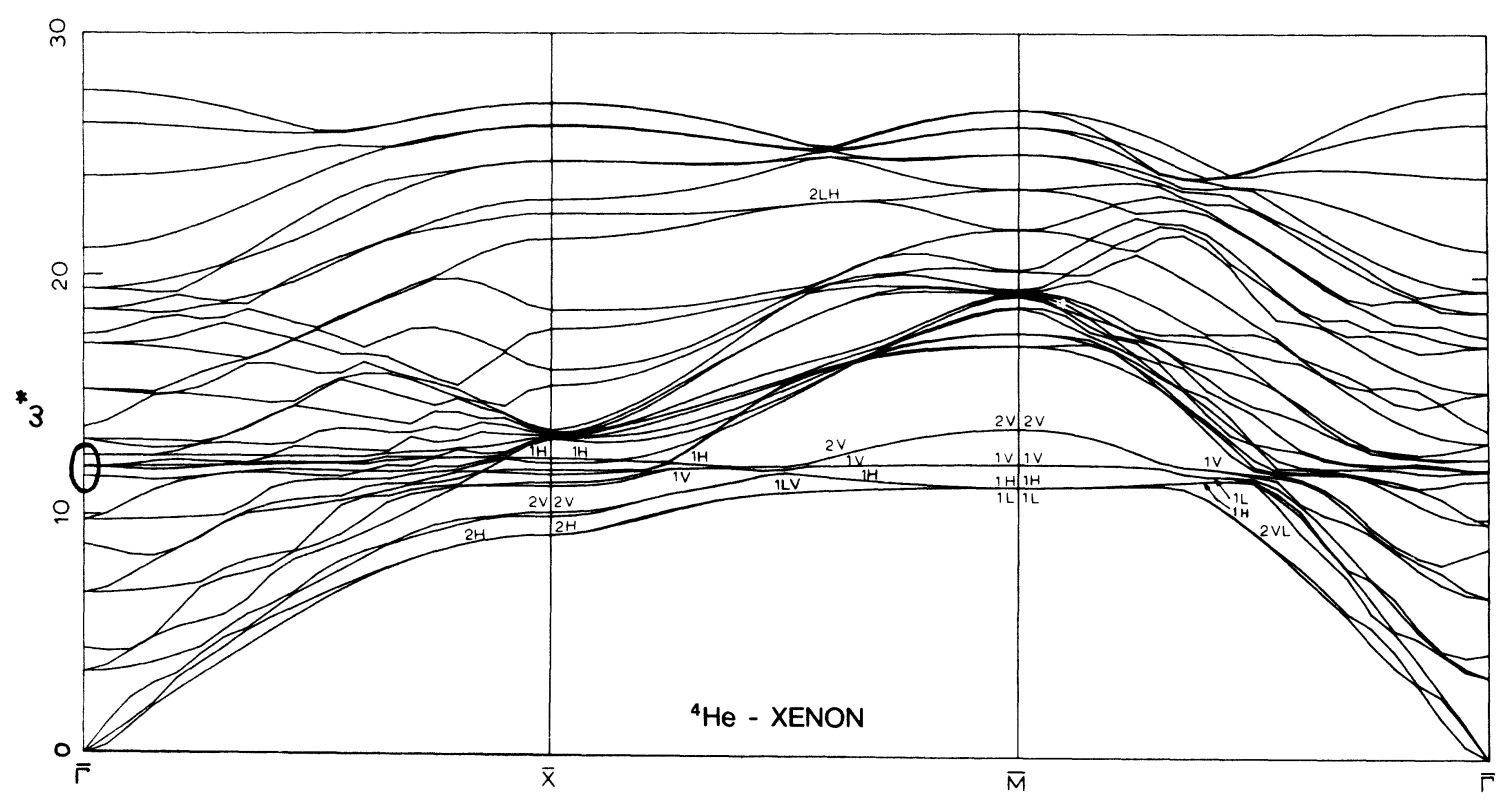

FIG. 1. Dimensionless frequency $\omega^{*}$ vs two-dimensional wave vector $\bar{q}$, along symmetry lines, for ${ }^{4} \mathrm{He}$ on Xe. There are nine substrate layers between two adsorbate layers, and thus $3 \times(9+2)=33$ modes per $\bar{q}$. 


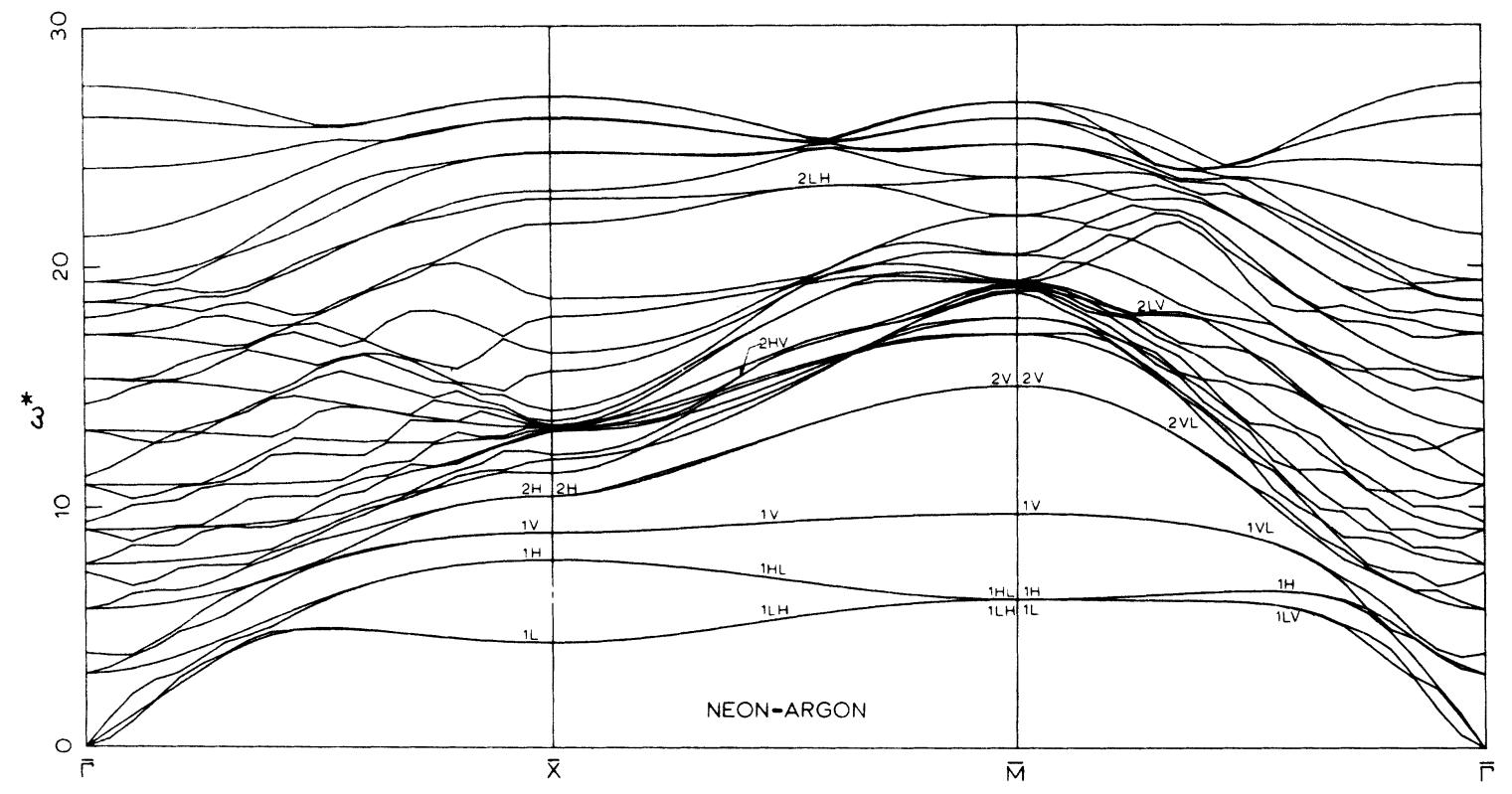

FIG. 2. $\omega^{*}$ vs $\bar{q}$ for $\mathrm{Ne}$ on Ar.

zation, parallel to the surface and transverse to the direction of propagation, and $V$ representing "vertical" polarization, transverse and perpendicular to the surface). For example, in Fig. 1, the mode labeled $2 L H$ along $\bar{X} \bar{M}$ has its maximum in the outermost layer of substrate atoms and is mostly longitudinal, with less transverse "horizontal" polarization and still less "vertical" polar-



FIG. 3. $\omega^{*}$ vs $\bar{q}$ for $\mathrm{Ne}$ on $\mathrm{Kr}$. 


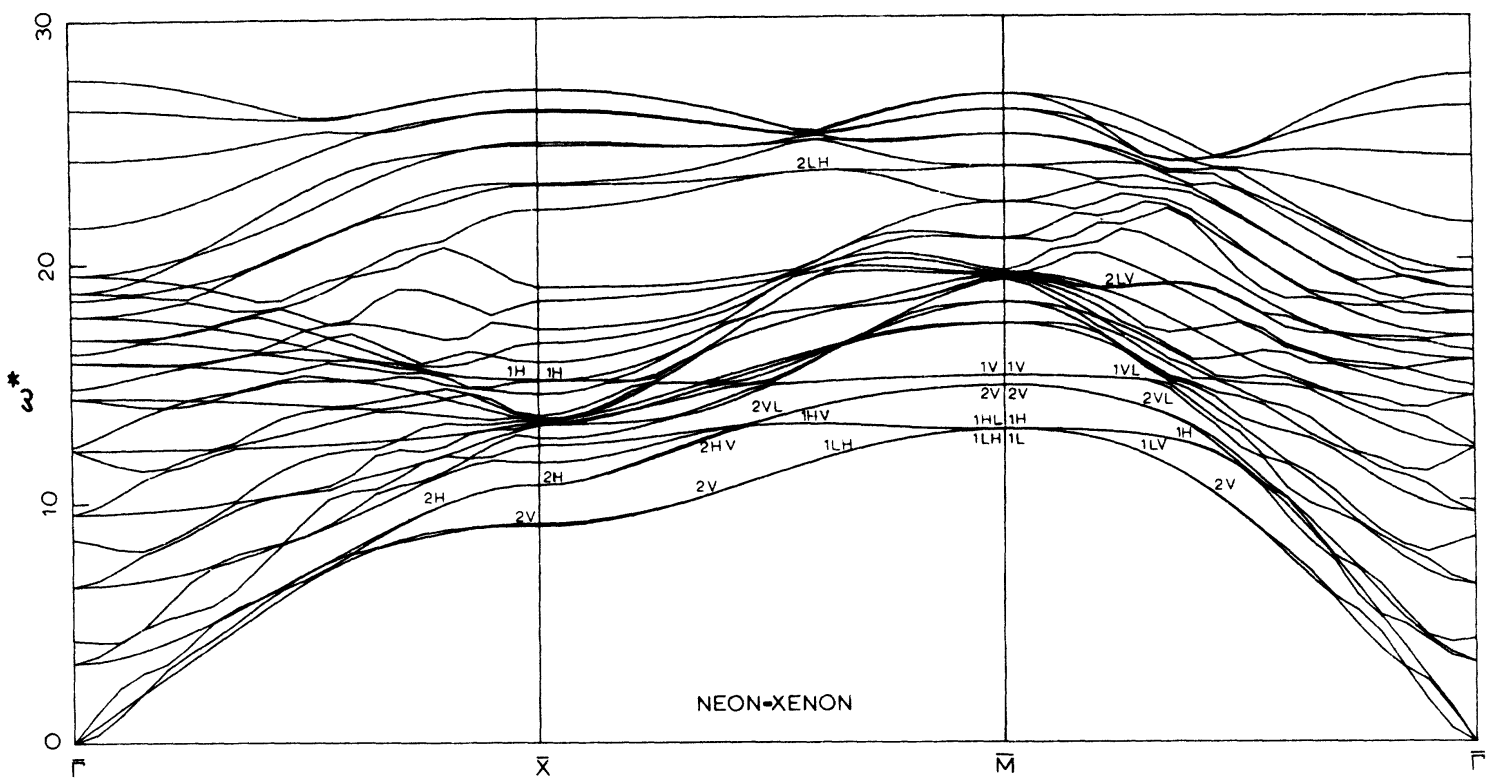

FIG. 4. $\omega^{*}$ vs $\bar{q}$ for $\mathrm{Ne}$ on $\mathrm{Xe}$.

ization. The amplitude and polarization of each mode in a given plane were determined by inspecting the associated eigenvector $\vec{\xi}(m)$. $^{3}$

\section{DISCUSSION OF RESULTS}

\section{A. ${ }^{4} \mathrm{He}$ on $\mathrm{Xe}$}

Because the He-Xe interaction is weak compared to the $\mathrm{Xe}-\mathrm{Xe}$ interaction, the surface modes for the clean Xe surface are largely unaffected by the He overlayer in Fig. 1. In particular, the mode labeled $2 H$ along $\bar{\Gamma} \bar{X}, 2 V$ near $\bar{M}$, and $2 V L$ on the right-hand side of $\bar{M} \bar{\Gamma}$ corresponds to $S_{1}$ of Allen et al. ${ }^{3}$ (see Fig. 10) for the clean Xe surface; and the mode labeled $2 \mathrm{~V}$ near $\bar{X}$ corresponds to $S_{4}$. There is, of course, hybridization of these modes with the new ones introduced by the He overlayer, between $\bar{X}$ and $\bar{M}$ and between $\bar{M}$ and $\bar{\Gamma}$. The mode

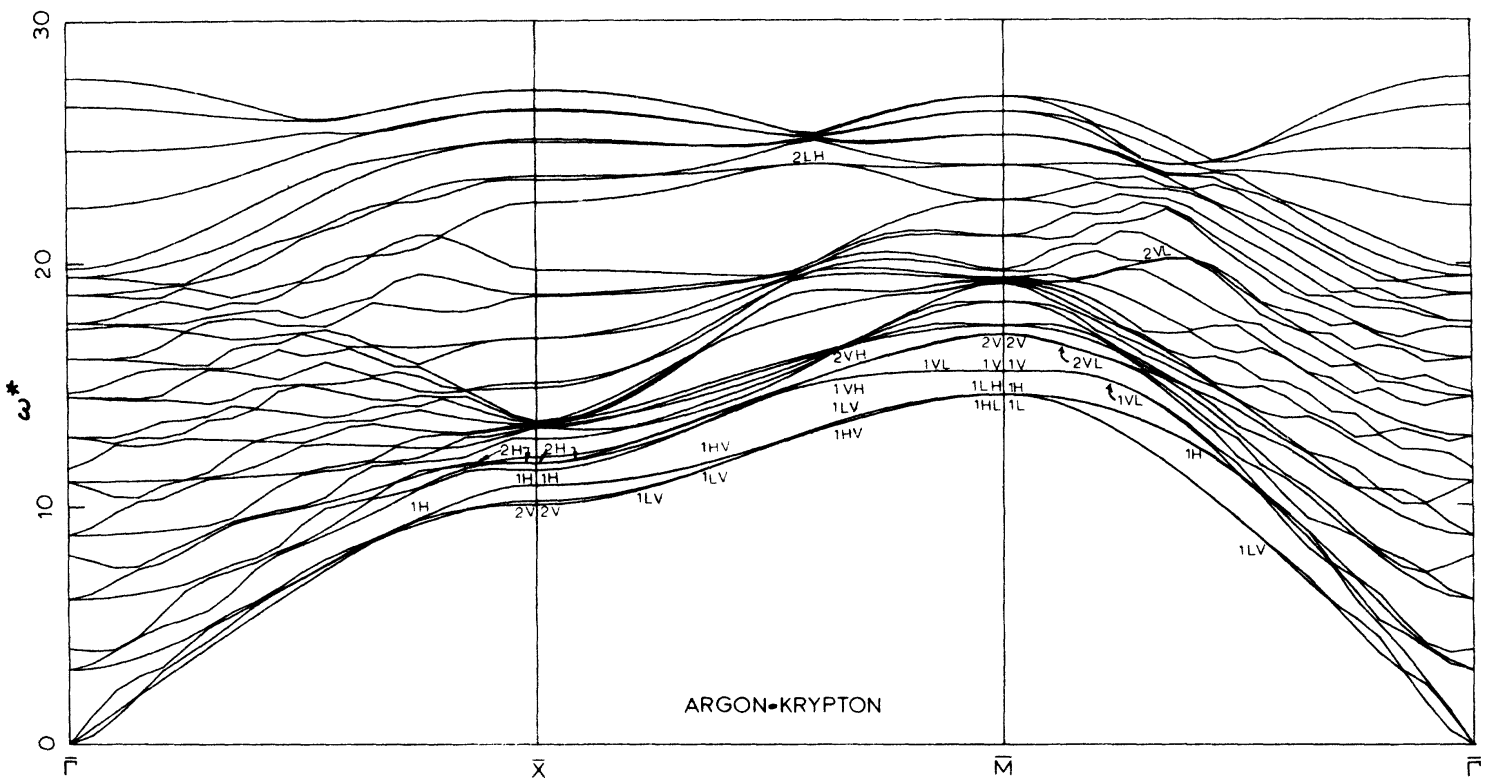

FIG. 5. $\omega^{*}$ vs $\bar{q}$ for $\mathrm{Ar}$ on $\mathrm{Kr}$. 


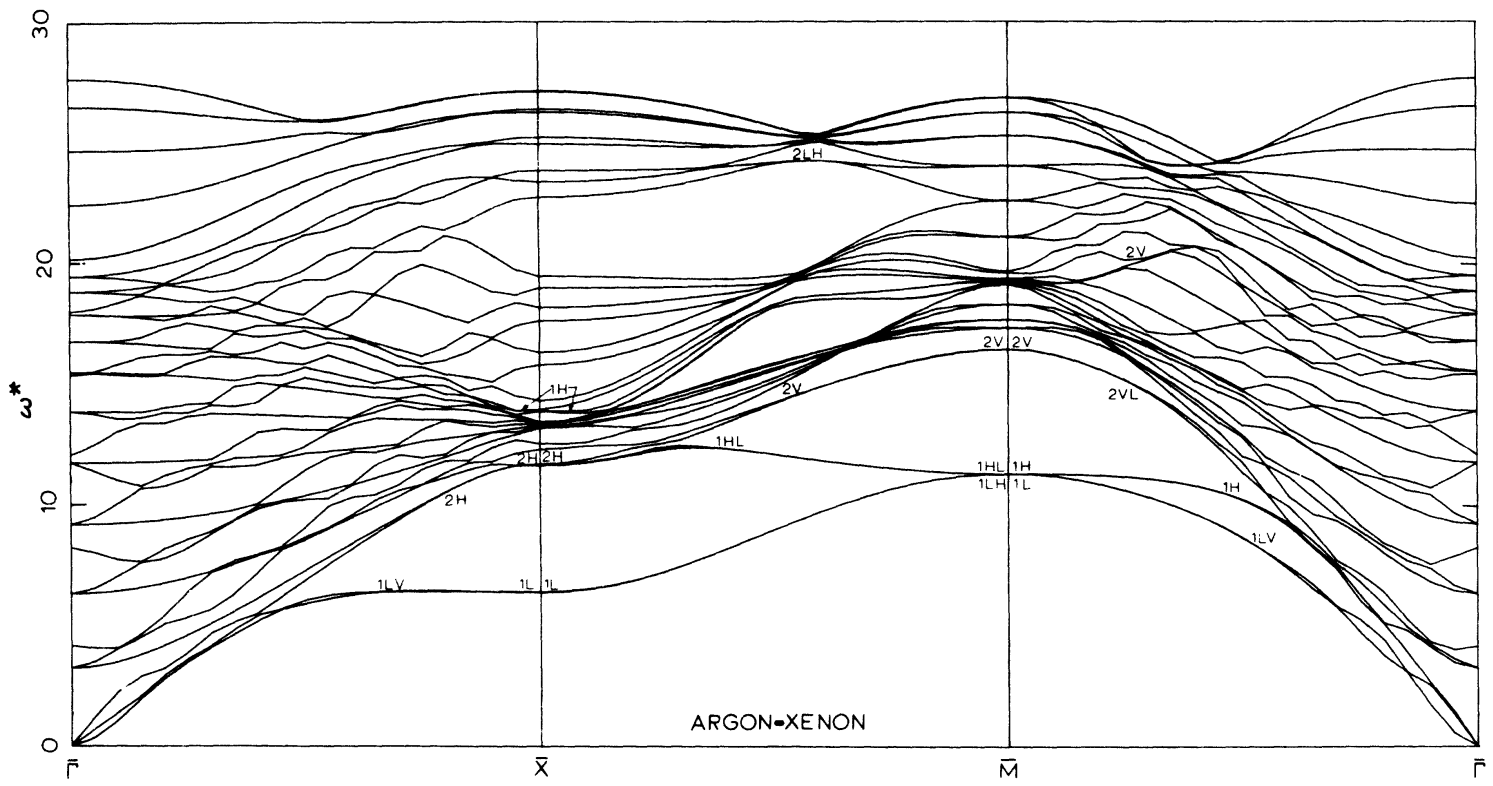

FIG. 6. $\omega^{*}$ vs $\bar{q}$ for $\mathrm{Ar}$ on $\mathrm{Xe}$.

labeled $2 L H$ along $\bar{X} \bar{M}$ corresponds to $S_{6}$ of Ref. 3 for the clean Xe surface.

In Fig. 8, a graph of the amplitude $|\vec{\xi}(m)|^{2}$ is shown for $1 H$ at $\bar{X}$. It is interesting that the modes associated with the He overlayer persist to $\bar{\Gamma}$ as resonances at $\omega^{*} \simeq 12$, surrounded by an oval curve in Fig. 1. Graphs of $|\vec{\xi}(m)|^{2}$ for these resonances are also given in Fig. 8.
B. Ne on $\mathrm{Ar}$

The lowest surface mode branches in Fig. 2 are very clear examples of the "principal" surface modes $^{2}$ associated with an adsorbate; there are three modes associated with the three directions of vibration $(L, H, V)$ for the adsorbate atoms. In addition, the branch labeled $2 H$ at $\bar{X}$ at $2 V$ at $\bar{M}$

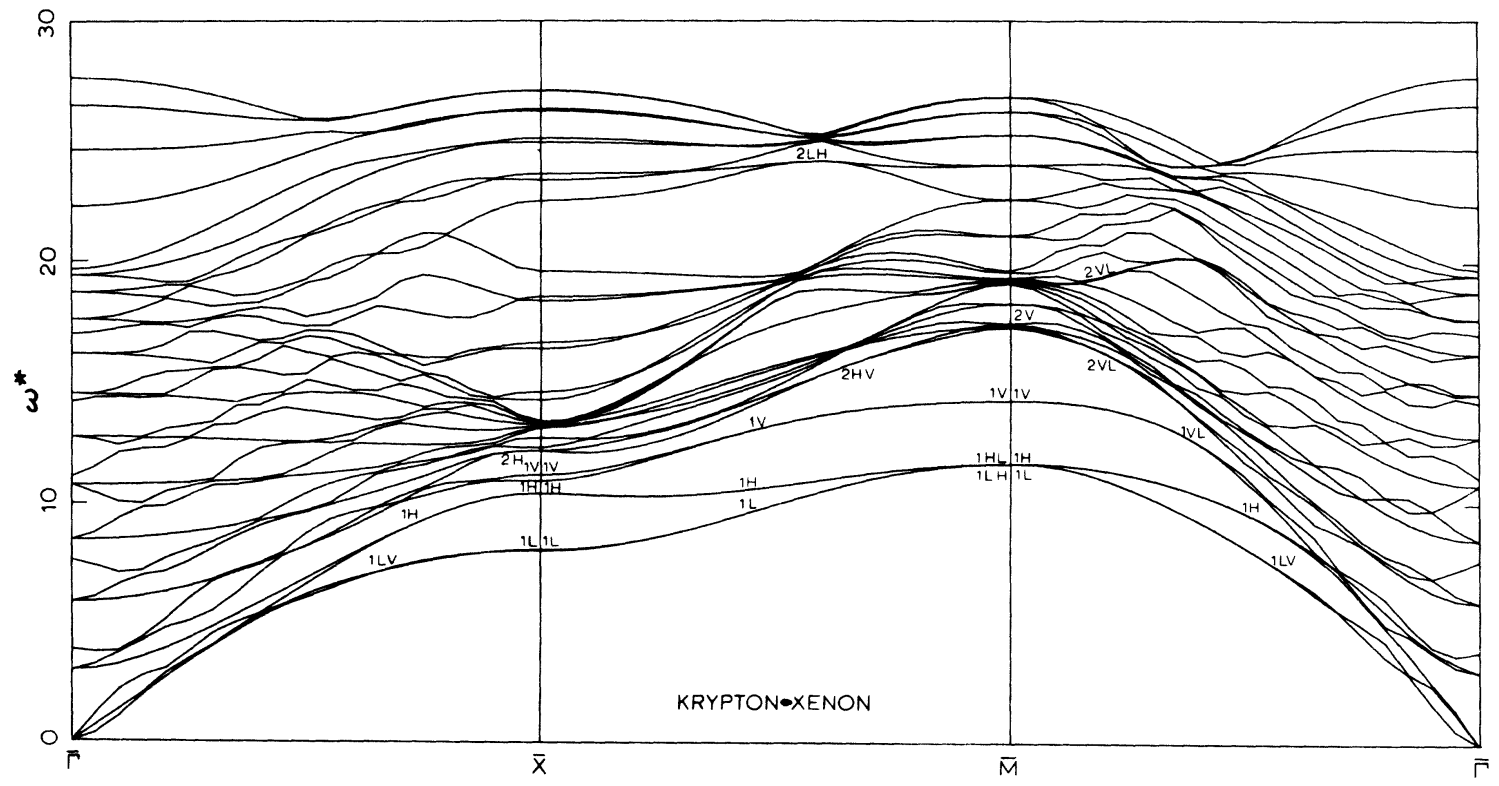

FIG. 7. $\omega^{*}$ vs $\bar{q}$ for $\mathrm{Kr}$ on $\mathrm{Xe}$. 


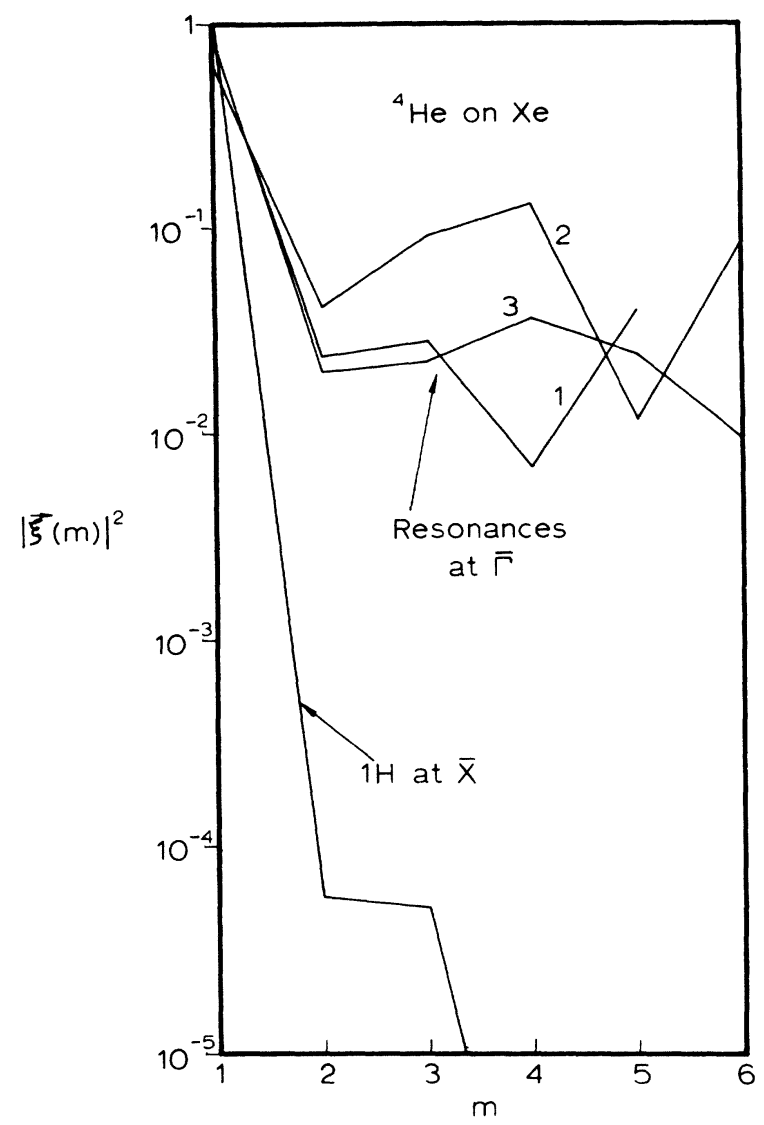

FIG. 8. $|\vec{\xi}(m)|^{2}$ vs $m$ for surface mode $1 H$ at $\bar{X}$ in Fig. 1, and for the resonances at $\bar{\Gamma}$ enclosed by an oval curve. Here $m=1$ for the layer of adsorbate atoms, $m=2$ for the first layer of substrate atoms, etc. " 1 " labels the highest pair of resonance modes, having inplane polarization; "2" labels a pair just below, also with in-plane polarization; and "3" labels the last (nondegenerate) pair with polarization perpendicular to the surface. These resonances are principally associated with vibrations of the adsorbed ${ }^{4} \mathrm{He}$ atoms.

clearly corresponds to $S_{1}$ of Ref. 3, Fig. 10 .

Figure 2 can be compared with Fig. 3 of Ref. 2, where the ordering of $1 \mathrm{~V}$ and the degenerate pair $1 L, 1 H$ at $\bar{M}$ is inverted. This comparison shows an interesting failure of the mass-defect model: In this model, the restoring force for in-plane vibrations of adsorbate atoms is larger than the restoring force for vibrations perpendicular to the surface, so the $1 \mathrm{~L}$ and $1 \mathrm{H}$ modes lie above the $1 \mathrm{~V}$ mode. In the present model, the restoring force for in-plane vibrations is smaller, because the $\mathrm{Ne}-\mathrm{Ne}$ interaction is weak compared to the $\mathrm{Ne}-\mathrm{Ar}$ interaction, and the $1 L$ and $1 H$ modes lie below the $1 \mathrm{~V}$ mode.

In comparisons with the mass-defect model, incidentally, one should regard the "mass" of a particle as really being a measure of the mass divided by an effective force constant; i.e., if $M_{a}$ and $M_{s}$ are the masses of adsorbate and substrate atoms in the mass-defect model, then we should take

$$
\frac{M_{a}}{M_{s}}=\frac{m_{a} / k_{a}}{m_{s} / k_{s}},
$$

where $m_{a}$ and $m_{s}$ are the real adsorbate and substrate atomic masses, and $k_{a}$ and $k_{s}$ are characteristic force constants for adsorbate and substrate. It is evident that in Fig. 2 the adsorbate is "heavier" than the substrate $\left(M_{a}>M_{s}\right)$ in this interpretation, because the weakness of the adsorbate interactions $\left(k_{a}<k_{s}\right)$ more than compensates for the lightness of the adsorbate atoms $\left(m_{a}<m_{s}\right)$.

\section{C. $\mathrm{Ne}$ on $\mathrm{Kr}$}

The three "principal" surface modes for the adsorbate are visible near $\bar{M}$ in Fig. 3 , although the frequencies of the principal modes are higher for $\mathrm{Ne}$ on $\mathrm{Kr}$ (relative to the bulk $\mathrm{Kr}$ spectrum) than they are for Ne on Ar (relative to the bulk Ar spectrum). The substrate mode $S_{1}$ is again evident, as $2 H$ near $\bar{X}$ and $2 V$ near $\bar{M}$, although it hybridizes twice with the adsorbate modes, $1 \mathrm{HV}$ and $1 V$, along $\bar{X} \bar{M}$. The mode labeled " 2 " is a second-layer mode with sagittal-plane polarization.

\section{D. $\mathrm{Ne}$ on $\mathrm{Xe}$}

The relative frequencies of the "principal" surface modes in Fig. 3 have again been raised, but the qualitative features are about the same as for $\mathrm{Ne}$ on $\mathrm{Kr}$. The mode labeled $2 \mathrm{~V}$ at $\bar{X}$ is almost as large in the first (adsorbate) layer as in the second (substrate) layer. The $1 H$ mode at $\bar{X}$ is a pure surface mode. Just to the right of $\bar{X}$ in Fig. 4 , this mode becomes a resonance, until it emerges from the bulk bands between $\bar{X}$ and $\bar{M}$. Plots of $|\vec{\xi}|^{2}$ for this mode are given in Fig. 9 for 11 evenly spaced points along $\bar{X} \bar{M}$, with point 1 being $\bar{X}$, point 2 just to the right of $\bar{X}$, etc., and point 11 being $\bar{M}$.

\section{E. Ar on $\mathrm{Kr}$}

The clean $\mathrm{Kr}$ surface mode $S_{1}$ is visible in Fig. 5 as $2 H$ at $\bar{X}$ and $2 V$ at $\bar{M}$. The three principal adsorbate modes exist at $\bar{M}$, as usual, and the lowest of these remain remarkably close together at $\bar{X}$ as $1 \mathrm{H}$ and $2 \mathrm{~V}$. As in the discussion of $\mathrm{Ne}$ on $\mathrm{Xe}$, just above, $2 \mathrm{~V}$ has a large amplitude in the adsorbate even though $|\vec{\xi}|^{2}$ reaches a maximum in the first substrate layer. This can be seen in Fig. 10 , where we have graphed $|\vec{\xi}|^{2}$ for the surface modes at $\bar{X}$ and $\bar{M}$. Graphs of the corresponding modes in Figs. 1-4, 6, and 7 show similar behavior. 


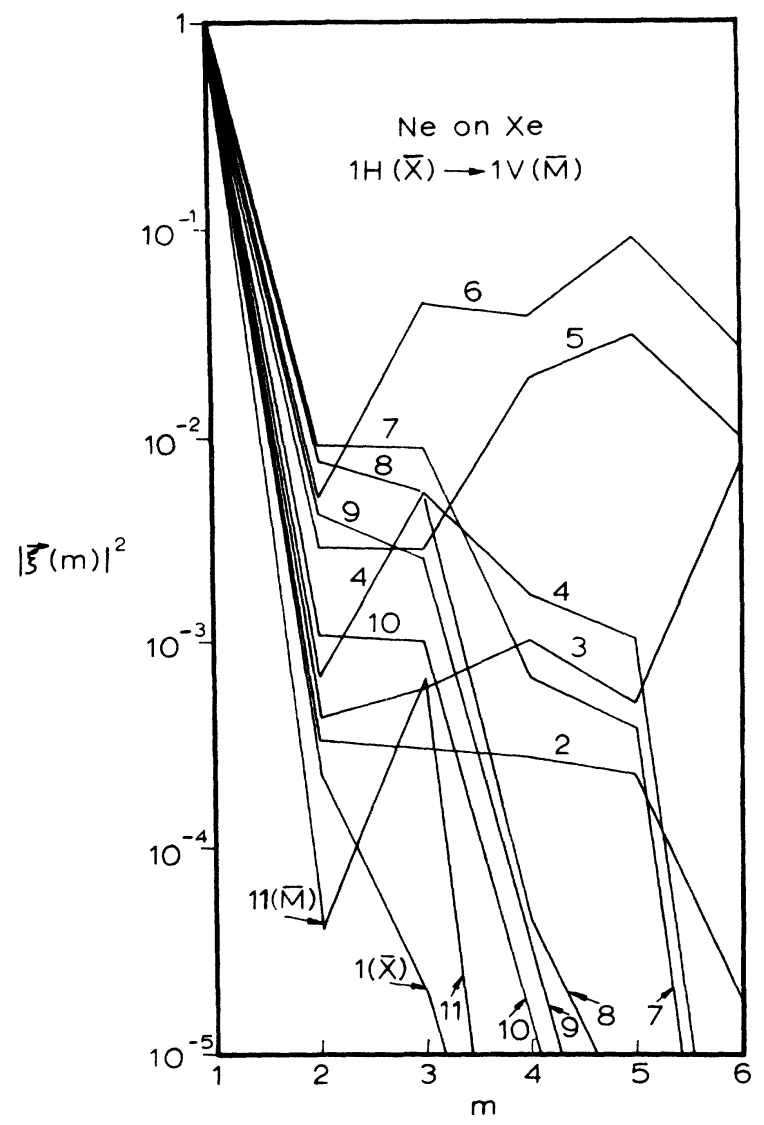

FIG. 9. $|\vec{\xi}(m)|^{2}$ vs $m$ for $1 H$ at $\bar{X}$ (point 1 ), $1 V$ at $\bar{M}$ (point 11), and a series of resonances and surface modes between these points along $\bar{X} \bar{M}$ in Fig. 4 .

\section{F. Ar on Xe}

There are no features in Fig. 6 that have not been discussed above.

\section{G. $\mathrm{Kr}$ on Xe}

All three principal adsorbate modes persist from $\bar{M}$ to $\bar{X}$. In this case, as for $\mathrm{Ar}$ on $\mathrm{Kr}$ and $\mathrm{Ar}$ on $\mathrm{Xe}$, the adsorbate-substrate interactions are strong enough to raise the substrate surface mode frequencies considerably.

\section{CONCLUSION}

The main surface modes are the three "principal" modes associated with the adsorbate, and

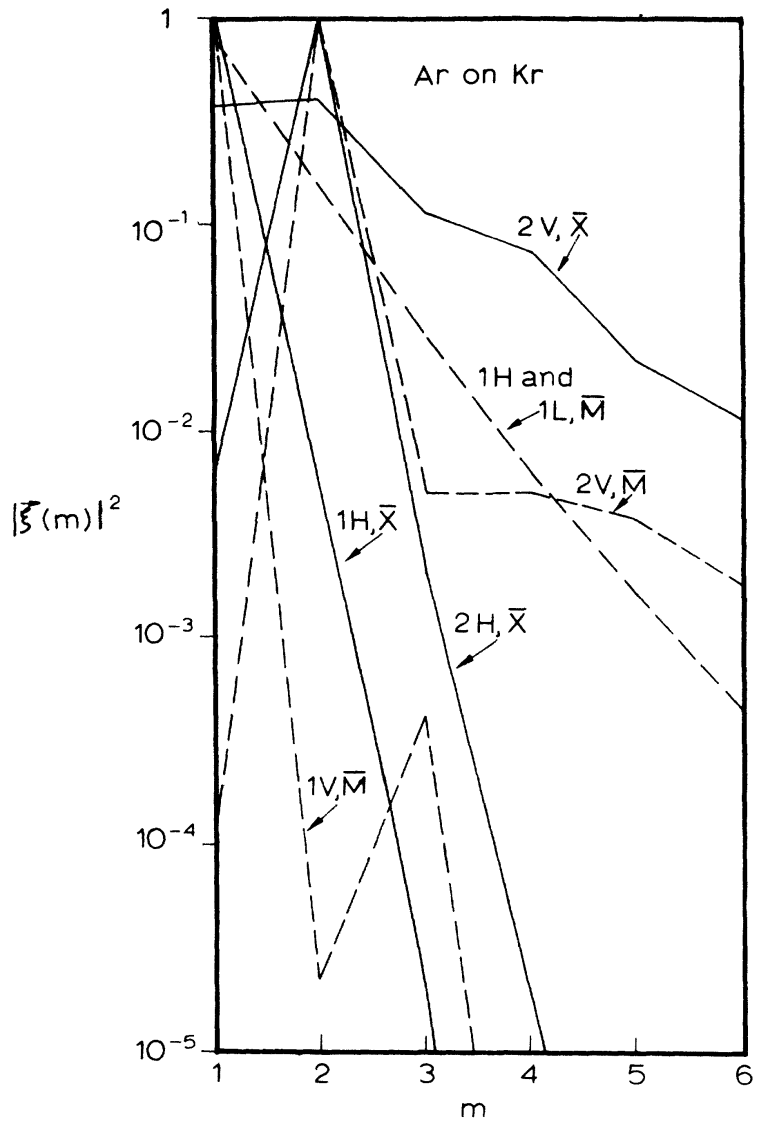

FIG. 10. $|\xi(m)|^{2}$ vs $m$ for surface modes at $\bar{X}$ and $\bar{M}$ in Fig. 5.

an $S_{1}$ surface mode for the clean-substrate material which persists (with its frequency changed) when the adsorbate is deposited.

The relative positions of the $1 L, 1 H$ modes and the $1 V$ mode at $\bar{M}$ (in Figs. 1-7) are inverted in the results for the "mass-defect" model, ${ }^{2}$ as discussed in Sec. III B. Also, in the present results for weakly interacting adsorbates ( $\mathrm{He}$ and $\mathrm{Ne}$ ), the substrate modes are much less affected by deposition of the adsorbate than in the results for the mass defect model. Both these facts show that the mass defect model can lead to qualitatively inaccurate conclusions about the vibrational modes of adsorbed atoms.
*Present address: School of Science and Technology, University of Houston at Clear Lake City, Clear Lake City, Tex.

${ }^{1}$ W. R. Lawrence and R. E. Allen, Phys. Rev. B (to be published).

${ }^{2}$ G. P. Alldredge, R. E. Allen, and F. W. de Wette, Phys. Rev. B $\underline{4}, 1682$ (1971). See also, L. Dobrzynski, Surf.
Sci. 20, 99 (1970); and L. Dobrzynski and D. L. Mills, J. Phys. Chem. Solids 30, 1043 (1969). However, G. Armand and J. B. Theeten [Phys. Rev. B 9, 3969 (1974)] included force-constant changes.

${ }^{3}$ R. E. Allen, G. P. Alldredge, and F. W. de Wette, Phys. Rev. B $\underline{4}, 1661$ (1971). 